Fizika Nizkikh Temperatur, 1999, v. 25, No 10, p. 1112-1115

Letters to the Editor

\title{
Rectification of surface polaritons in an insulator in a magnetic field at the boundary with a metal
}

\author{
I. E. Chupis and D. A. Mamaluy \\ B. Verkin Institute for Low Temperature Physics and Engineering, National Academy of Sciences of Ukraine \\ 47 Lenin Ave., 310164, Kharkov, Ukraine \\ E-mail: mamaluy@ilt.kharkov.ua \\ Received May 11, 1999
}

\begin{abstract}
Surface polaritons in a semi-infinite insulator in a constant magnetic field at the boundary with an ideal metal or a superconductor have been considered. These polaritons are induced by dynamic magnetoelectric interaction, which exists in any insulator in the presence of a magnetic field. The modes of these surface polaritons differ appreciably in opposite directions of the magnetic field or the propagation of the wave. As a result polaritons of the given optical or IR frequency propagate only in one direction, which is the effect of rectification of surface electromagnetic waves. The inversion of the magnetic field results in «switching on» or «switching off» of surface polaritons.
\end{abstract}

PACS: $\mathbf{7 5 . 8 0 . + q}, 78.20 . \mathrm{Jq}$

The influence of the magnetic field on the surface polaritons in metals and semiconductors is the subject of a wide range of theoretical and experimental studies. A similar investigation of insulators has also been started [1]. In metals and semiconductors polaritons are considered as a result of interaction of electromagnetic waves with free electrons of a crystal (plasmon polaritons). In the presence of a magnetic field these polaritons are called magnetoplasmon polaritons (see, for example, Ref. 2). In insulators the interaction of optical phonons with a magnetic field can be described by dynamic magnetoelectric energy [3], which takes into account the electron and ion contributions to the electric polarization.

It is well known that in the presence of a magnetic field the polariton spectrum is nonreciprocal, i.e., $\omega(-\mathbf{k}) \neq \omega(\mathbf{k})$. The nonreciprocity in the polariton spectrum is a weak effect.

In this paper we show strong nonreciprocity of surface polaritons in a semi-infinite insulator at its boundary with an ideal metal or a superconductor in the presence of a magnetic field. The modes of polaritons propagating in opposite directions essentially differ. Surface polaritons with a given frequency propagate only in one direction with respect to the magnetic field: rectification of surface polari- tons. The inversion of the wave vector $\mathbf{k} \rightarrow-\mathbf{k}$ corresponds to that of the magnetic field.

Let us consider a uniaxial insulator ( $z$ is an easy axis), although the results obtained are of general importance.

We start with the density of insulator energy $W$ used earlier for the investigation of surface polaritons in an insulator in an electric field [4]:

$$
W=\frac{{ }^{c}}{2} P_{z}^{2}+\frac{{ }^{c_{2}}}{2}\left(P_{x}^{2}+P_{y}^{2}\right)+\frac{\Pi^{2}}{2 \rho}-\mathbf{e P}+\xi \mathbf{P}[\Pi \times \mathbf{H}],
$$

where $\mathbf{P}$ is the electric polarization; $\Pi$ is the momentum density; $\mathbf{H}=\mathbf{H}_{0}+\mathbf{h}$; and $\mathbf{e}$ and $\mathbf{h}$ are alternating electric and magnetic fields. The uniform external magnetic field $\mathbf{H}_{0}$ is imposed in the $y$ direction. In (1) we disregard the spatial dispersion of the electric polarization. The last term in (1) corresponds to the dynamic magnetoelectric energy [3]. This is the energy of the interaction $\mathbf{P}$ with an effective electric field $\mathbf{E}_{\text {ef }}=-1 / c[\mathbf{v} \times \mathbf{H}]$ produced by the motion of charge $e$ with velocity $\mathbf{v}$ in a magnetic field ( $c$ is the speed of light). Since the momentum $\Pi=m \mathbf{v} / V_{0}, \xi=V_{0} / m c$ and the constant $\rho$ in kinetic energy $\Pi^{2} / 2 \rho$ is $\rho=m / V_{0}$, where $m$ is the mass of the charge and $V_{0}$ is the unit cell volume. 
In general, the electric polarization $\mathbf{P}$ consists of ion and electron parts. In the IR region of the spectrum the contribution of ions to the magnetoelectric energy is prevalent; then $m$ is the ion mass and $\Pi$ is the ion momentum. In the optical region of the spectrum the electron contribution to the polarization is rather ionic contribution, $m$ is the electron mass and $\Pi$ is the electron momentum. The dynamic magnetoelectric energy [the last term in Eq. (1)] is a scalar; therefore, it is present in the energy of any crystal.

The linear response of an insulator in the field of an electromagnetic wave in the absence of damping can be obtained in the same way as in Ref. 3. In our case the components of the dielectric constant $\varepsilon_{i k}$ are

$$
\begin{gathered}
\varepsilon_{1}=\varepsilon_{x x}=1+\frac{4 \pi \bar{\omega}_{0}^{2}\left(\omega_{e}^{2}-g^{2} H_{0}^{2}-\omega^{2}\right)}{\left(\omega^{2}-\omega_{1}^{2}\right)\left(\omega^{2}-\omega_{2}^{2}\right)}, \\
\varepsilon_{2}=\varepsilon_{y y}=1+\frac{4 \pi \bar{\omega}_{0}^{2}}{\omega_{0}^{2}-\omega^{2}}, \\
\varepsilon^{\prime}=i \varepsilon_{x z}=i\left(\varepsilon_{z x}\right)^{*}=\frac{8 \pi \omega g H_{0} \bar{\omega}_{0}^{2}}{\left(\omega^{2}-\omega_{1}^{2}\right)\left(\omega^{2}-\omega_{2}^{2}\right)}, \\
\varepsilon=\varepsilon_{z z}=\frac{\left(\omega^{2}-\Omega_{1}^{2}\right)\left(\omega^{2}-\Omega_{2}^{2}\right)}{\left(\omega^{2}-\omega_{1}^{2}\right)\left(\omega^{2}-\omega_{2}^{2}\right)} .
\end{gathered}
$$

Here

$$
\begin{gathered}
\omega_{1,2}^{2}=\frac{1}{2}\left[\omega_{0}^{2}+\omega_{e}^{2}+2 g^{2} H_{0}^{2} \mp\right. \\
\left.\mp \sqrt{\left(\omega_{0}^{2}-\omega_{e}^{2}\right)^{2}+8 g^{2} H_{0}^{2}\left(\omega_{0}^{2}+\omega_{e}^{2}\right)}\right], \\
\Omega_{1,2}^{2}=\frac{1}{2}\left[\omega_{0}^{2}+\Omega_{e}^{2}+2 g^{2} H_{0}^{2} \pm\right. \\
\left. \pm \sqrt{\left(\omega_{0}^{2}-\Omega_{e}^{2}\right)^{2}+8 g^{2} H_{0}^{2}\left(\omega_{0}^{2}+\Omega_{e}^{2}\right)}\right] \\
g=\frac{e}{m c}, \bar{\omega}_{0}^{2}=\frac{e^{2}}{m V_{0}}, \omega_{0}=\bar{\omega}_{0} \sqrt{c_{2}}, \omega_{e}=\bar{\omega}_{0} \sqrt{c_{1}}, \\
\Omega_{e}^{2}=\omega_{e}^{2}+4 \pi \bar{\omega}_{0}^{2} .
\end{gathered}
$$

The frequency $\omega_{e}$ is the excitation frequency of electric polarization $\mathbf{P}$ along the easy axis $z$, and $\omega_{0}$ is the excitation frequency of the transverse components of polarization $P_{x}, P_{y}$ in the absence of a magnetic field. For a uniaxial crystal we have

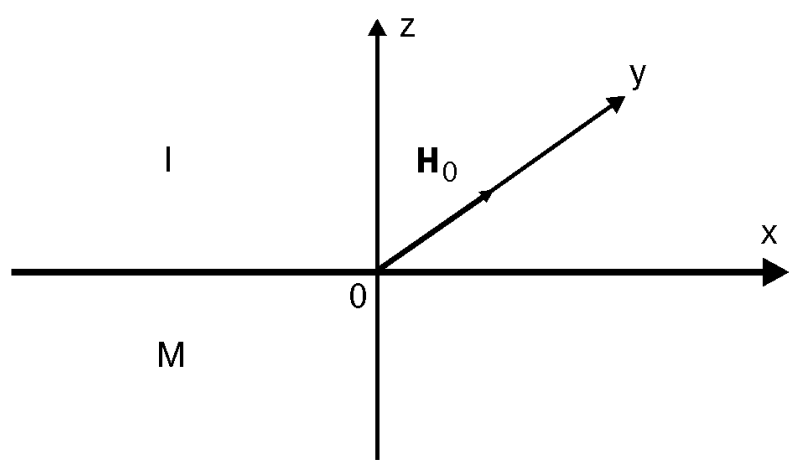

Fig. 1. The insulator (I) is in semi-infinite space $(z>0)$ and the metal $(\mathrm{M})$ is in the space where $z<0$. The magnetic field $\mathbf{H}_{0}$ is in the positive direction of the $y$ axis in the contact plane; the wave vector $\mathbf{k}$ is along the $x$ axis.

$\omega_{e}<\omega_{0}$. In a constant magnetic field these frequencies $\omega_{e}$ and $\omega_{0}$ become $\omega_{1}$ and $\omega_{2}$, respectively.

The nondiagonal components of the dielectric constants $\varepsilon_{x z}$ and $\varepsilon_{z x}$ in Eq. (2), which indicate the presence of gyrotropy, are proportional to the first degree of the magnetic field.

The geometry we consider is shown in Fig. 1. A semi-infinite insulator $(z>0)$ is in contact with an ideal metal $z<0$. A static magnetic field $\mathbf{H}_{0}$ is applied along the $y$ axis parallel to the interface. The boundary conditions at $z=0$ are

$$
b_{z}=\tilde{b}_{z}, d_{z}=\tilde{d}_{z}, \mathbf{e}_{t}=\tilde{\mathbf{e}}_{t}=0, \mathbf{h}_{t}=\tilde{\mathbf{h}}_{t},
$$

where $\mathbf{b}$ and $\mathbf{d}$ are the magnetic and the electric inductions; $\mathbf{e}_{t}$ and $\mathbf{h}_{t}$ are the tangential fields; and the letters with a tilde refer to a metal. In the case of a superconductor $\tilde{b}_{z}=0$. We assume that magnetic permeability of an insulator is $\mu=1$.

The solution of the Maxwell's equations for surface polaritons that propagate along the $x$ axis is

$$
\mathbf{e}, \mathbf{h} \propto \exp \left[i\left(k_{x} x-\omega t\right)-k_{0} z\right], k_{0}>0, z>0 .
$$

Here $k_{0}^{-1}$ is the depth of penetration of the field into an insulator.

Using Eqs. (2), (4), and (5), we can write the Maxwell's equations in the form

$$
h_{y}=-\frac{c k_{x}}{\omega} e_{z}, h_{y}=-\frac{\omega}{c k_{x}} \varepsilon e_{z},\left(k_{0}-\frac{\omega^{2}}{c^{2}} \frac{\varepsilon^{\prime}}{k_{x}}\right) e_{z}=0 \text {. }
$$

Only $e_{z}$ and $h_{y}$ differ from zero in the wave; in this case the depth of their penetration and the dispersion relation are

$$
k_{0}=\frac{\omega^{2}}{c^{2}} \frac{\varepsilon^{\prime}(\omega)}{k_{x}}= \pm \frac{\omega}{c} \frac{\varepsilon^{\prime}(\omega)}{\sqrt{\varepsilon(\omega)}}, \quad k_{x}^{2}=\frac{\omega^{2}}{c^{2}} \varepsilon(\omega) .
$$




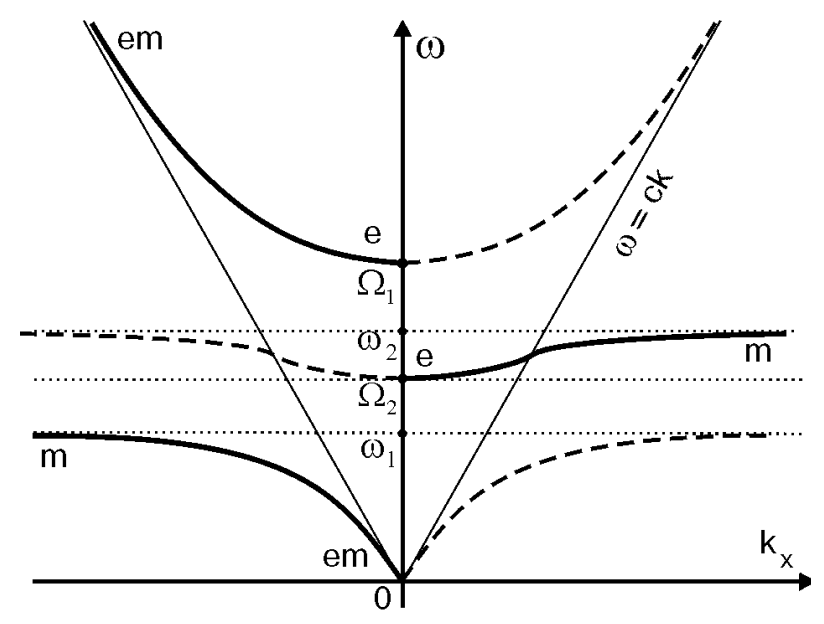

Fig. 2. Modes of surface polaritons. The thick solid curves correspond to the case where $\mathbf{H}_{0} \uparrow \uparrow y$ in the optical region and to the case where $\mathbf{H}_{0} \uparrow \downarrow y$ in the IR region. The dashed curves are surface modes for the opposite direction of $\mathbf{H}_{0}$.

In our case $b_{z}=h_{z}=0$; therefore, all the results are also valid when the insulator is in contact with a superconductor.

Taking into account the necessary conditions $k_{0}>0, \quad \varepsilon>0$, expression (2), and the ratio $\Omega_{e}>\omega_{0}$, which is natural for a uniaxial crystal, we obtain the polariton modes, which are shown in Fig. 2. For ion excitations (the IR region of the spectrum) the gyromagnetic ratio $g>0$ and Fig. 2 corresponds to the case $H_{0}<0$. For electron excitations (the optical region) $g<0$ and Fig. 2 corresponds to the case $H_{0}>0$. The thick solid curves in Fig. 2 are modes of the surface polaritons $\left(k_{0}>0\right)$. The dashed curves correspond to «unphysical» excitations, which increase exponentially inside the insulator $\left(k_{0}<0\right)$. All surface polariton excitations are real. Two left thick solid curves in Fig. 2 are modes of the polaritons that run to the left. The surface polaritons that run to the right have one mode (thick solid curve of $k_{x}>0$ ). The substitution of $k_{x}$ by $-k_{x}$ in Fig. 2 corresponds to the inversion of the magnetic field, $H_{0} \rightarrow-H_{0}$. In this case the dashed curves are the modes of surface polaritons and the thick solid curves correspond to «unphysical» excitations.

The ratio of amplitudes of the electric and magnetic fields in the wave is

$$
\left|\frac{e_{z}}{h_{y}}\right|=\left[\frac{\left(\omega^{2}-\omega_{1}^{2}\right)\left(\omega^{2}-\omega_{2}^{2}\right)}{\left(\omega^{2}-\Omega_{1}^{2}\right)\left(\omega^{2}-\Omega_{2}^{2}\right)}\right]^{1 / 2} .
$$

We see from (8) that the magnetic field dominates in excitations near the frequencies $\omega_{1}$ and $\omega_{2}$ $\left(e_{z} \rightarrow 0\right)$. The electric field dominates near the

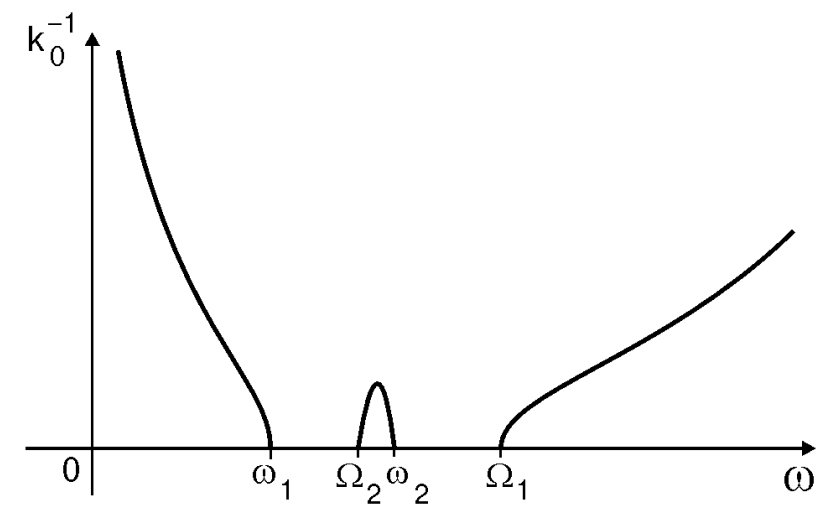

Fig. 3. The frequency dependences of the polariton penetration depths into the insulator.

frequencies $\Omega_{1}$ and $\Omega_{2}\left(h_{y} \rightarrow 0\right)$. In Fig. 2 the letters $e, m$ and $e m$ denote the excitation character; i.e., $e-$ electrical, $m-$ magnetic, $e m-$ electromagnetic.

The frequency range $\left[\Omega_{2}, \omega_{2}\right]$ for the polaritons running to the right (Fig. 2) is very small, $\omega_{2}-\Omega_{2} \sim g^{2} H_{0}^{2} \omega_{0}^{-1}$. Since the gyromagnetic ratio $g=e / m c$ for electrons is on the order of $10^{7}$ and $\omega_{0} \sim 10^{14}-10^{15} \mathrm{rad} / \mathrm{s}$, for the magnetic fields $H_{0} \sim 10^{4}$ Gs $\left(\omega_{2}-\Omega_{2}\right)$ is on the order of $10^{7}-10^{8}$.

When the magnetic field $H_{0}$ decreases, the frequency range $\left[\Omega_{2}, \omega_{2}\right]$ tends to zero, and the magnitude $k_{0} \propto \varepsilon^{\prime} \propto H_{0}$ also tends to zero, i.e., the depth of penetration $k_{0}^{-1}$ tends to infinity. In the limit $H_{0}=0$, the frequency $\omega_{2}=\Omega_{2}$ and «unphysical» polaritons (the dashed curves in Fig. 2) are real; i.e., in the absence of magnetic field there are bulk polaritons with two symmetric excitation branches (the lower and the upper curve in Fig. 2), for which $\omega(-\mathbf{k})=\omega(\mathbf{k})$. Thus, in the absence of a constant magnetic field only the bulk polaritons exist in the system, which corresponds to the known results [2]. It is also clear from the last equation in (6) that when $\varepsilon^{\prime}=0\left(H_{0}=0\right), k_{0}$ must be equal to zero; i.e., the depth of penetration $k_{0}^{-1}=\infty$.

As to the constant magnetic field $\left(\varepsilon^{\prime} \neq 0\right)$, in its presence the polaritons are surface polaritons. The depth of their penetration, $k_{0}^{-1} \propto H_{0}^{-1}$, decreases with increasing magnetic field. Figure 3 shows the frequency dependence of the depth of penetration of polaritons into the insulator.

In summary, in the presence of a constant magnetic field the surface polaritons exist in a semi-infinite insulator, which is in contact with an ideal metal or a superconductor. The depth of penetration of their field into the insulator is inversely proportional to the magnetic field. The surface modes are strongly nonreciprocal with respect to the propagation direction, $\omega(\mathbf{k}) \neq \omega(-\mathbf{k})$. The number of modes for waves propagating in opposite directions is dif- 
ferent. Since the modes are not close to each other, excitations with the given frequency propagate only in one direction with respect to the magnetic field. The surface electromagnetic waves can therefore be rectified in the system under consideration. The inversion of the magnetic field is equal to that of the propagation direction. Thus, the inversion of the magnetic field results in «switching on» or «switching off» of surface polaritons with a given frequency. Only surface polaritons exist in the presence of a constant magnetic field.

We wish to thank V. I. Fomin and V. S. Kurnosov for useful discussions.
1. I. E. Chupis and N. Ya. Alexandrova, J. Korean Phys. Soc. 32, 51134 (1998).

2. V. M. Agranovich and D. L. Mills (eds.), Surface Polaritons, North-Holland, Amsterdam (1982).

3. I. E. Chupis, Ferroelectrics 204, 173 (1997).

4. I. E. Chupis and D. A. Mamaluy, Pis'ma Zh. Eksp. Teor. Fiz. 68, 876 (1998) [JETP Letters 68, 922 (1998)]. 